Document downloaded from:

http://hdl.handle.net/10251/146289

This paper must be cited as:

Min, R.; Marques, C.; Bang, O.; Ortega Tamarit, B. (03-2). Moiré phase-shifted fiber Bragg gratings in polymer optical fibers. Optical Fiber Technology. 41:78-81.

https://doi.org/10.1016/j.yofte.2018.01.003

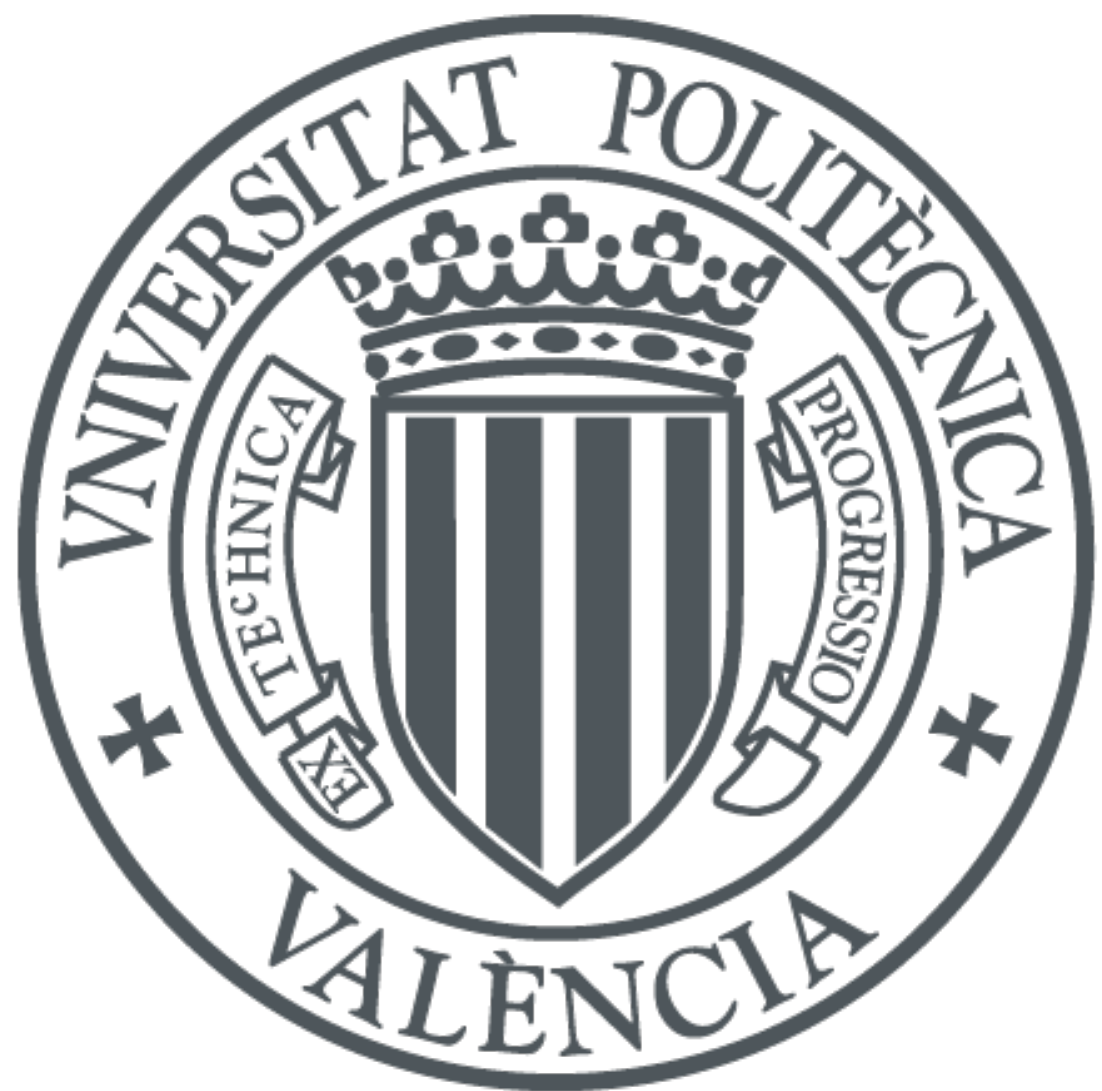

The final publication is available at

https://doi.org/10.1016/j.yofte.2018.01.003

Copyright Elsevier

Additional Information 


\section{Moiré Phase-Shifted Fiber Bragg Gratings in Polymer Optical Fibers}

Rui Min ${ }^{1}$, Carlos Marques ${ }^{2}$, Ole Bang ${ }^{3}$ and Beatriz Ortega ${ }^{1 *}$

${ }^{1}$ ITEAM Research Institute, Universitat Politècnica de València, Valencia, Spain

${ }^{2}$ Instituto de Telecomunicações and Physics Department \& I3N, Universidade de Aveiro, Portugal

${ }^{3}$ DTU Fotonik, Department of Photonics Engineering, Technical University of Denmark, Denmark

E-mail: bortega@dcom.upv.es

We demonstrate a phase-shifted fiber Bragg grating in polymer optical fibers as a narrowband transmission filter for a variety of applications at telecom wavelengths. The filters have been fabricated by overlapping two uniform fiber Bragg gratings with slightly different periods to create a Moiré grating with only two UV laser pulses (one pulse is 15 ns). Experimental characterization of the filter is provided under different conditions and the strain and temperature sensitivities have been measured. 
Phase-shifted fiber Bragg gratings (PS-FBG) in silica fibers have been demonstrated during the last decade as attractive very narrow filters for a variety of applications such as real-time monitoring, switching and demodulating. ${ }^{1-2)}$ Very recently published papers report PS-FBG applications also in the microwave photonics field, such as tunable bandstop-to-bandpass microwave photonic filters, which opens new perspectives for these devices. $^{3)}$

It is well-known that a narrowband transmission filter can be obtained from an FBG when a single phase shift is introduced inside the grating whose location and magnitude can be adjusted according to the desired transmission band. Moreover, multiple phase shifts can be used to tailor the transmission spectrum for wavelength demultiplexing in DWDM applications ${ }^{4)}$

Polymer optical fibers (POFs) have several advantages over silica fibers, such as a larger elongation before breakage, a higher thermo-optic coefficient, and a lower Young's modulus. These advantages will provide a broad tuning range either straining or heating the fiber, and easy handling due to its low stiffness and therefore, lower installations cost. Although the product of bandwidth and channel length is somewhat reduced due to the higher loss, ${ }^{5)}$ POFs are already extensively used in buildings, cars, trains, airplanes and other important short-range applications. Recently, the introduction of the new polymer called CYTOP ${ }^{6}$ with reduced losses at telecom wavelengths has led to new promising applications for POFs.

FBGs in silica fibers is a very mature filtering technology both for telecommunication and sensors ${ }^{7)}$ since a large variety of high performance FBGs can be fabricated by creating a UV light induced periodic variation in the refractive index of the fiber core. Since the first polymer FBG was reported in $1999^{8)}$, several promising devices have been demonstrated, such as embedded POF FBG sensors ${ }^{9)}$, microstructured POF (mPOF) sensors at $827 \mathrm{~nm}^{10}$ ), Fabry-Perot cavity based on polymer FBG as refractive index sensor ${ }^{11)}$, FBGs in low-loss multi-mode CYTOP POFs ${ }^{12)}$, and mPOF FBG sensors in the polymer TOPAS ${ }^{13)}$, which is humidity insensitive. Despite of this, POF and mPOF gratings are still under research. Fast high quality FBG fabrication requires highly photosensitive fibers; so far great advances have been done in doping POFs ${ }^{14)}$. Furthermore, previous work ${ }^{15)}$ demons trates that grating time stability can be correctly addressed by proper fiber annealing.

High resolution applications require the fabrication of polymer FBGs with sharp 
characteristics which, in combination with the large sensitivity of polymers, will lead to a new generation of accurately tunable devices. Therefore, the fabrication of phase-shifted gratings in polymer optical fiber is identified as one of the challenges for the development of novel sensors or communication subsystems. However, the literature only provides a single report on a phase-shifted FBG (PS-FBG) in POFs, which was for use at THz frequencies ${ }^{16)}$ by using a point-by-point FBG fabrication method. In terms of minimizing fabrication times of gratings, a $248 \mathrm{~nm}$ UV laser was employed to fabricate FBGs in benzyl dimethyl ketal (BDK) doped fiber with a single UV laser pulse ${ }^{17}$.

In this letter, to the best of our knowledge, we report the first PS-FBG in POFs fabricated with the phase-mask technique. Here we use two gratings with slight different periods, which have been superimposed in the fiber in order to forma Moiré structure with a $\pi$ phase shift in the center of the device ${ }^{18)}$. A very narrowband transmission filter is achieved, and strain and temperature sensitivities are provided.

A PS-FBG consists of a grating where a discrete phase shift is inserted at a certain point inside the grating. Punctual modification of the refractive index of the fiber core or relative spatial shift of the fiber with respect to the phase mask, when using phase-mask based grating fabrication, are alternatives to fabricating a PS-FBG, although both require very high spatial resolution equipment. However, the phase-shifted structure can be reproduced as a Moiré grating, where two gratings of equal amplitude but different periods, $\Lambda_{1}$ and $\Lambda_{2}$, are superimposed ${ }^{18)}$. This structure has one rapidly and one slowly varying envelope, which periods are $\Lambda_{\mathrm{s}}$ and $\Lambda_{\mathrm{c}}$, respectively, as shown in Fig. 1(a) and there is an intrinsic $\pi$ phase-shift at the crossover point. According to the theory, other phase shift values can be achieved by super-imposing gratings with different amplitudes and multiple phase-shifts can be obtained either by using longer gratings or by changing $\Lambda_{1}$ or $\Lambda_{2}$ while the grating length is maintained.

In our setup, a $248 \mathrm{~nm}$ Coherent Bragg Star Industrial-LN krypton fluoride (KrF) emitting an output pulse power of $2.5 \mathrm{~mJ}$ energy and $15 \mathrm{~ns}$ duration was employed for grating inscription. A slit was employed to control the width of the beam. The fiber was mounted horizontally in between two v-grooves with $0.1 \%$ strain and the beam was focused onto the fiber using a plano-convex cylindrical lens (Newport CSX200 AR.10) (see inset of Fig. 1(b)). A mPOF made of PMMA with a three ring hexagonal cladding 
microstructure (see Fig. 1 (b) has a hole-to-pitch ratio of 0.47 with an average hole diameter of $1.74 \mu \mathrm{m}$ and an average pitch of $3.70 \mu \mathrm{m}{ }^{19)}$, which makes it close to endlessly single-mode. The fiber core is doped with BDK to increase the photosensitivity to $\mathrm{UV}^{19}$ ). Before use the fiber, it was annealed for 24 hours at $70^{\circ} \mathrm{C}$. The UV light was passed through a $567.80 \mathrm{~nm}$ pitch phase mask over a $2.5 \mathrm{~mm}$ fiber length. Initially, a first uniform grating with a period of $289.4 \mathrm{~nm}$ was fabricated with one pulse shown in Fig. 2. The fiber was manually connectorised with around $2 \mathrm{~dB}$ insertion losses per connector. An Optical Spectrum Analyzer (YOKAGAWA AQ6373B) was employed to measure the grating spectral response with $0.01 \mathrm{~nm}$ resolution bandwidth. According to Fig. 1 (a), and provided the grating length $L_{0}=2.5 \mathrm{~mm}$ is equal to $\Lambda_{c}$ and the fiber length between two flexure stage accessories L is $202 \mathrm{~mm}$, the required pitch of the second grating can be calculated:

$$
\Lambda_{2}=\frac{\mathrm{L}_{\mathrm{o}} \Lambda_{1}}{\mathrm{~L}_{\mathrm{o}}-2 \Lambda_{1}}=289.466 \mathrm{~nm}
$$

Therefore, the second grating fabrication requires the fiber strained up to a total length of $\mathrm{L}+\Delta \mathrm{L}$, which can be calculated as follows:

$$
\Delta \mathrm{L}=\frac{\Lambda_{2}-\Lambda_{1}}{\Lambda_{1}} * \mathrm{~L}=0.0459 \mathrm{~mm}
$$

Therefore, since both gratings are spatially superimposed, a Moiré grating is obtained and a phase shift is formed inside ${ }^{18)}$.

Fig. 2 shows the reflected signal of the first uniform grating and also when the second grating is overlapped with two pulses irradiation, where a phase shift has appeared. The new opened reflection $3 \mathrm{~dB}$ band is $0.035 \mathrm{~nm}$. Repetitive experimental results confirm that a PS-FBG can be easily achieved by this method.

The simulation of the response of a single phase shifted grating can be easily done by inserting a phase shift matrix using Matrix method ${ }^{20)}$. Fig. 3 shows the theoretical and experimental PS-FBG transmission and reflectivity response. The full width at half-maximum of the FBG stopband is about $0.1 \mathrm{~nm}$ and, as a result of the phase-shift, a sub-picometer transmission band appears with $3 \mathrm{~dB}$ insertion losses. The PS-FBG reflectivity of both peaks (\#1 and \#2), separated by the notch is higher than $80 \%$. The experimental results fit well with the simulation resul ts where the slight difference could be due to any non-homogeneity from UV laser beam, imperfections in the POF manufacturing, or even the real grating length inscribed to be less than the phase mask length ${ }^{21)}$. 
As explained above, this method can be easily employed to obtain multiple phase shifted gratings by changing $\Lambda_{2}$ (see eqs. (1) and (2)). Fig. 4 depicts the reflectivity response of a PS-FBG based on the Moiré structure resulting from with $\Delta \mathrm{L}=0.0705 \mathrm{~mm}$, which corresponds to $\Lambda_{2}=289.501 \mathrm{~nm}\left(\Lambda_{\mathrm{c}}=1.875 \mathrm{~mm}\right)$, according to eq. (2) and therefore two phase shifts are encountered along the $2.5 \mathrm{~mm}$ long grating, as expected from Fig. 1. Furthermore, the experiment also confirmed three phase shifts when the second overlapped grating is written using $\Delta \mathrm{L}=0.0936 \mathrm{~mm}$, which results to $\Lambda_{2}=289.534 \mathrm{~nm}\left(\Lambda_{\mathrm{c}}=1.25\right.$ $\mathrm{mm})$.

In this section, the fabricated PS-FBGs were tested under different strain and temperature. The PS-FBG was placed into a translation stage to strain the fiber step-wise in time. The evolution of wavelength and reflectivity for both peaks and notch of the PS-FBG reflectivity spectrum was monitored when the strain was changed from 0 to $1.1 \%$ every 5 min at room temperature, as depicted in Fig. 5 (a). A wavelength shift of $7.32 \mathrm{~nm}$ was observed with respect to the unstrained grating, which indicates a linear strain sensitivity of $0.708 \pm 0.002 \mathrm{pm} / \mu \varepsilon$ for both peaks and notch (see Fig. 5 (b)), which is similar with previous uniform FBGs in annealed POFs ${ }^{15}$ ). Fig 5 (c) shows the reflected power increases slightly with strain as expected for uniform polymer FBGs for this range of strain ${ }^{22}$.

Temperature sensitivity is measured with other fabricated PS-FBG to avoid the effect of strain measurement. The grating was put on a Peltier plate which contains a small v-groove and the temperature was controlled by a temperature electronic controller. Some silicone grease was placed on the fiber to increase the temperature conduction. We measure wavelength and reflected power response of the grating from $25^{\circ} \mathrm{C}$ to $55^{\circ} \mathrm{C}$ every $5 \mathrm{~min}$. The total wavelength shifts about $2.00 \mathrm{~nm}$ with temperature increase from $25^{\circ} \mathrm{C}$ to $55^{\circ} \mathrm{C}$. The achieved temperature sensitivity was about $-0.069 \pm 0.001 \mathrm{~nm} /{ }^{\circ} \mathrm{C}$, after fitting to a linear model. It can be seen that a $2 \mathrm{~nm}$ change can be achieved with a temperature variation of only $30^{\circ} \mathrm{C}$, which is larger than the attained in silica PS-FBG by several hundreds degree temperature variation $\left(\sim 10.3 \mathrm{pm} /{ }^{\circ} \mathrm{C}\right){ }^{23)}$. Also, this measured temperature sensitivity is similar to the values already reported for uniform FBGs in the same polymer fiber ${ }^{24)}$.

In conclusion, for the first time to our knowledge we demonstrate the fabrication of a PS-FBG in polymer optical fibers using the phase mask technique. We obtained the phase 
shift by superimposing two quasi similar uniform fiber Bragg gratings to form a Moiré structure by using two UV pulses irradiation. Furthermore, the flexibility of such technique has been shown by fabricating gratings with multiple phase shifts. POFs are very attractive for the deployment of current and future high bandwidth access networks and the phase shifted devices open new opportunities for applications in WDM networks and also for microwave photonic applications. Strain and temperature sensitivity have been measured as suitable for future highly tunable devices, which will be developed based on easy handling and low cost materials provided stress relaxation issues and related stability are correctly addressed.

\section{Acknowledgments}

This work was supported by Fundação para a Ciência e Tecnologia (FCT)/MEC through national funds and when applicable co-funded by FEDER - PT2020 partnership agreement under the projects UID/EEA/50008/2013, and UID/CTM/50025/2013. C. A. F. Marques also acknowledges the financial support from FCT through the fellowship SFRH/BPD/109458/2015. Rui Min acknowledges the financial support from the China Scholarship Council (201506680061). The research leading to these results has also received funding from the Marie Curie Actions of the European Union's 7th Framework Programme FP7/2007-2013/ under REA grant agreement No. 608382. We are grateful to Dr. David Sáez-Rodríguez for providing the polymer optical fibre used in this work. 


\section{References}

1) N.F.Naim, M.S.Ab-Rahman, H.A. Bakarman, A.A. Bakar, IEEE/OSA J. of Opt. Comm. and Netw.5, 1425-1430 (2013).

2) T.Y.Kim, M.Hanawa, S.J.Kim, S.Hann, Y.H.Kim, W.T.Han, C.S.Park, IEEE Photon. Technol. Lett., 18, 1834 - 1836, (2006).

3) X.Han, J.Yao, J. Lightw. Technol., 33, 5135-5139 (2015).

4) G.P.Agrawal, S.Radjic, IEEE Photon. Technol. Lett.6, 995-997 (1994).

5) M.Joncic, R.Kruglov, M.Haupt, R.Caspary, J.Vinogradov, U.H.P.Fischer, IEEE Photon. Technol. Lett.26, 1328-1331 (2014).

6) Y.Koike, T.1shigure, ECOC97, pp22-25 September 1997.

7) K.M.Chung, L.Dong, C.Lu, H.Y Tam, Opt. Express.19, 12664-12672 (2011).

8) Z. Xiong, G.D.Peng, B.Wu, P.L.Chu, IEEE Photon. Technol. Lett.11, 352-354(1999).

9) G.Rajan, M.Ramakrishnan, Y.Semenova, E.Ambikairajah, G.Farrell, G.D. Peng, J.Lightware. Technol. 32, 1726-1733 (2014).

10) I. P. Johnson, K. Kalli, D. J. Webb, Electron. Lett. 46, 1217-1218 (2010).

11) M. F. Ferreira, G.Statkiewicz-Barabach, D.Kowal, P. Mergo, W. Urbanczyk, O.\& Frazão, Opt. Commun., 394, 37-40 (2017).

12) A. Lacraz, M. Polis, A. Theodosiou, C. Koutsides, K. Kalli, IEEE Photon. Technol. Lett. 27, 693-696, (2015).

13) W. Yuan, L. Khan, D.J. Webb, K. Kalli, H.K. Rasmussen, A. Stefani, O. Bang, Opt. Express 19, 19731-19739 (2011)

14) Y.Luo, Q.Zhang, H.Liu, G.D.Peng, Opt.Lett. 35,751-753 (2010).

15) A.Pospori, C. A. F. Marques, D.Sáez-Rodríguez, K.Nielsen, O. Bang, \& D. J. Webb, Optical Fiber Technology, 36, 68-74(2017).

16) S.F.Zhou, L.Reekie, Y.T.Chow, H.P.Chan, K.M.Luk, IEEE Photon. Technol. Lett., 24, 1875-1877 (2012).

17) A.Pospori, C. A. F.Marques, O. Bang, D. J.Webb, \& P. André, Optics Express, 25, 9028-9038 (2017).

18) D.C.J.Reid, C.M.Ragdale, I.Bennion, D.J.Robbins, J.Buus, W.J.Stewart, Electron. Lett.26, 10-12 (1990).

19) D.Sáez-Rodríguez, K.Nielsen, O.Bang, D.J.Webb, Opt.Lett. 38, 3769-3772 (2013). 
20) T.Erdogan. J. Lightwave Technol, 15, 1277-1294(1997).

21) C. A. F. Marques, PhD Thesis, Universidade de Aveiro, (2013). (available in: https://ria.ua.pt/handle/10773/12218)

22) W. Yuan, A. Stefani, M. Bache, T. Jacobsen, B. Rose, N. Herholdt-Rasmussen, F. K. Nielsen, S. Andresen, O. B. Sørensen, K. S. Hansen, and O. Bang, Opt. Commun. 284, 176-182 (2011).

23) S. F. O.Silva, L. A. Ferreira, F. M.Araujo, J. L. Santos, O.\& Frazão. Fiber and Integrated Optics, 30, 9-28(2011).

24) C. Marques, A. Pospori, D. Sáez-Rodríguez, K. Nielsen, O. Bang, D. J. Webb, , Proc. SPIE 9634, 24th International Conference on Optical Fibre Sensors, 96345V-96345V-4 (2015). 


\section{Figure Captions}

Fig. 1. a) Moiré grating structure. b) Experimental setup for FBG fabrication, inset: Transverse structure of the mPOF,

Fig. 2. Reflected power of uniform grating and phase shifted grating.

Fig.3.Experimental (dashed blue line) and simulations (solid red line) of the spectral response of the PS-FBG: (a) transmission, (b) reflectivity

Fig. 4. Reflected power of multiple phase shifted FBGs (solid line, two PS-FBGs, dashed line, three PS-FBGs).

Fig. 5. (a) Reflected spectra under different strain. (b) Central wavelength changes and (c) maximum reflected power for both peaks (\#1 and \#2) and notch of the PS-FBG under different strain.

Fig. 6. Central wavelength of the PS-FBG under different temperatures. 
(a)

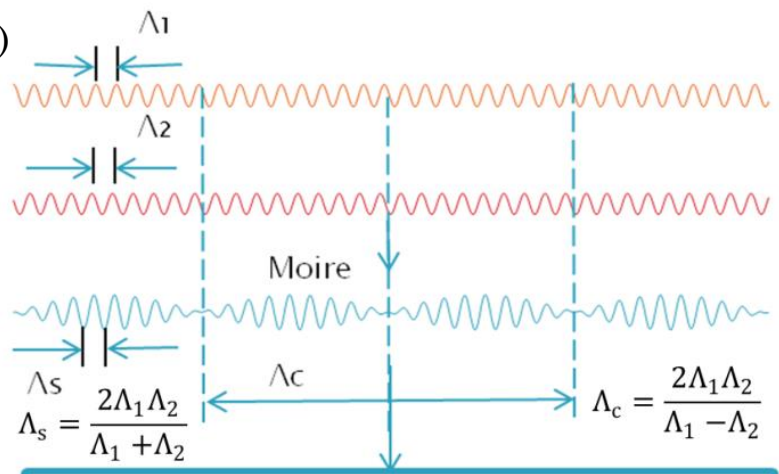

(b)

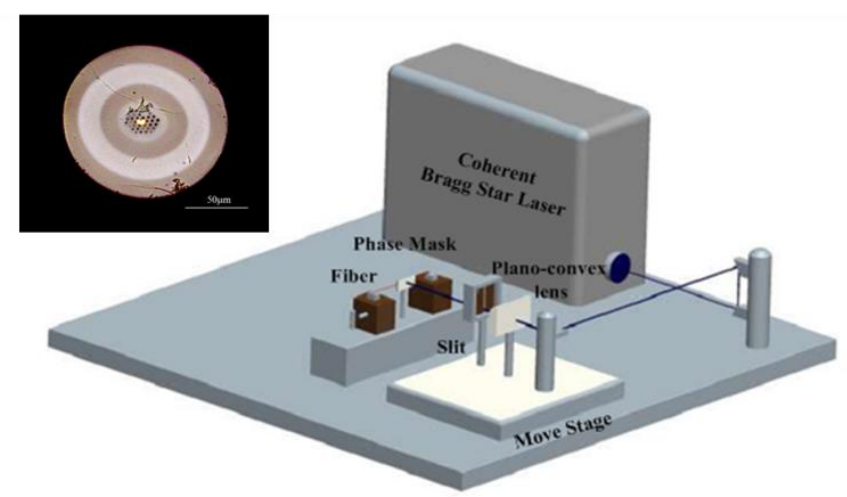

Fig.1.

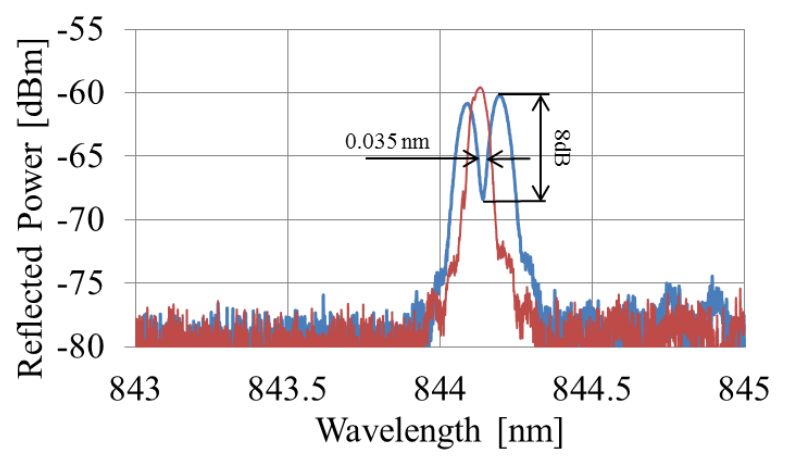

Fig. 2. 

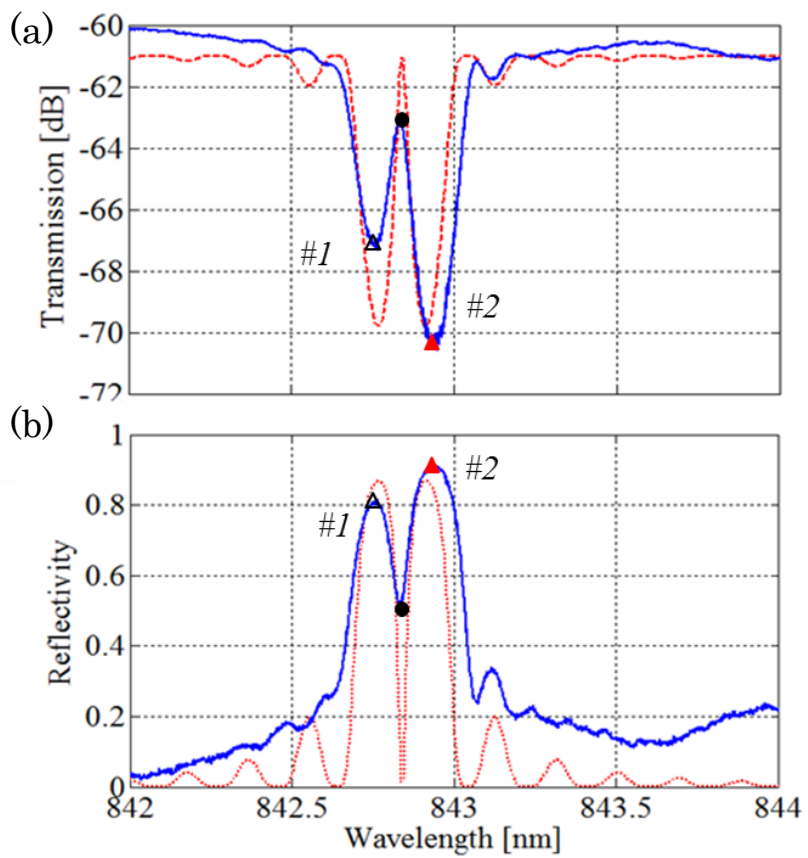

Fig. 3.

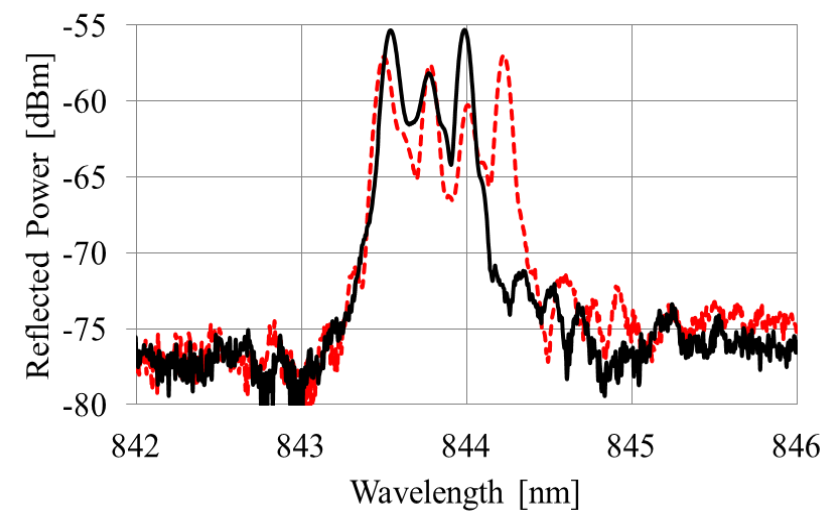

Fig. 4. 

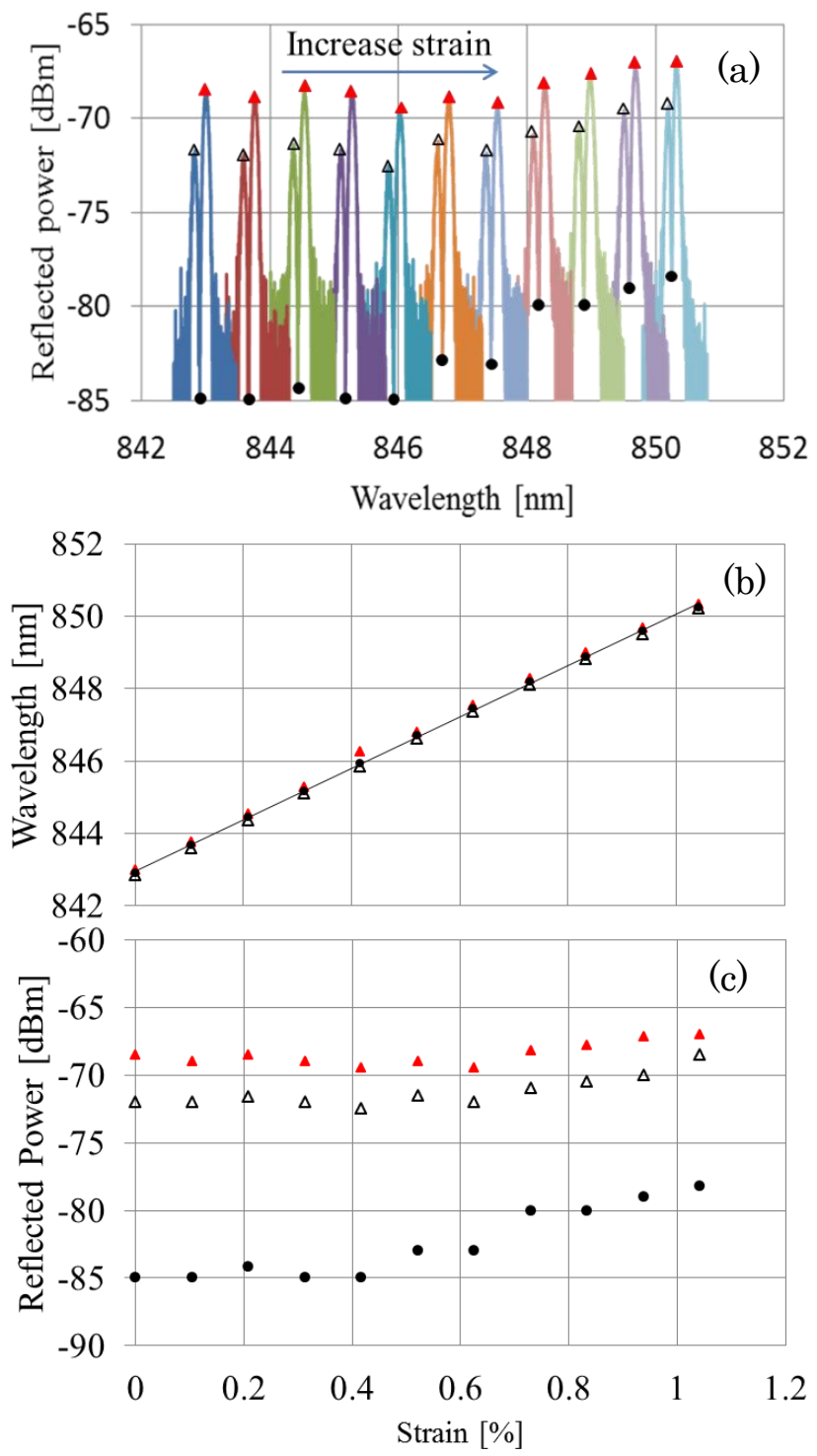

Fig.5. 


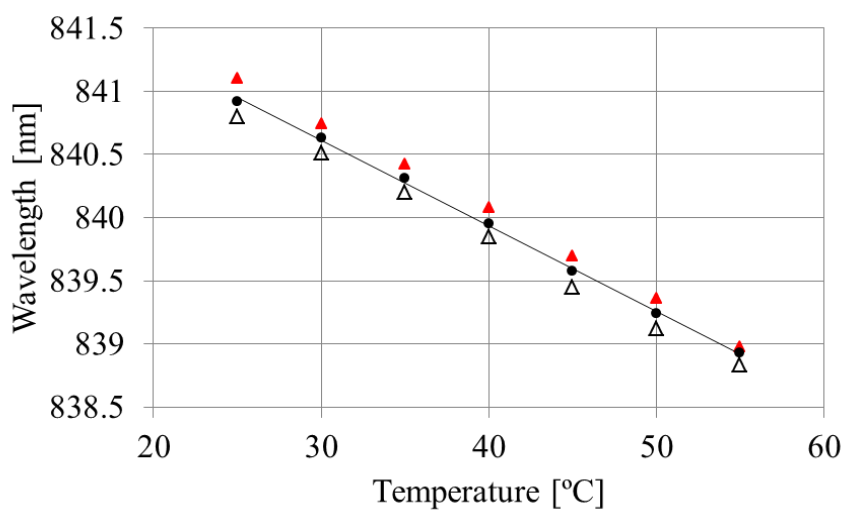

Fig.6. 